Corresponding author: dbabovic@mayo.edu

(C) 2019 Zepeda-Mendoza et al. This article is distributed under the terms of the Creative Commons Attribution-

NonCommercial License, which permits reuse and redistribution, except for commercial purposes, provided that the original author and source are credited.

Ontology terms: bulbous nose; cone-shaped epiphyses of phalanges 2 to 5 ; depigmentation/

hyperpigmentation of skin; long philtrum; prominent ear helix; short stature; thick eyebrow

Published by Cold Spring Harbor Laboratory Press

doi:10.1101/mcs.a004655

\section{An intragenic duplication of TRPS1 leading to abnormal transcripts and causing trichorhinophalangeal syndrome type I}

\author{
Cinthya J. Zepeda-Mendoza, ${ }^{1,6}$ Margot A. Cousin, 2,3,6 Shubham Basu, ${ }^{3}$ \\ Garrett Jenkinson, ${ }^{3}$ Gavin Oliver, ${ }^{3}$ Siobhan T. Pittock, ${ }^{4}$ Linda B. Baughn, ${ }^{1}$ \\ Eric W. Klee, ${ }^{1,2,3,5}$ and Dusica Babovic-Vuksanovic ${ }^{1,4,5}$
}

\footnotetext{
${ }^{1}$ Department of Laboratory Medicine and Pathology, ${ }^{2}$ Center for Individualized Medicine, ${ }^{3}$ Department of Health Sciences Research, ${ }^{4}$ Department of Pediatrics and Adolescent Medicine, ${ }^{5}$ Department of Clinical Genomics, Mayo Clinic, Rochester, Minnesota 55905, USA
}

\begin{abstract}
Trichorhinophalangeal syndrome type I (TRPSI) is a rare disorder that causes distinctive ectodermal, facial, and skeletal features affecting the hair (tricho-), nose (rhino-), and fingers and toes (phalangeal) and is inherited in an autosomal dominant pattern. TRPSI is caused by loss of function variants in TRPS1, involved in the regulation of chondrocyte and perichondrium development. Pathogenic variants in TRPS1 include missense mutations and deletions with variable breakpoints, with only a single instance of an intragenic duplication reported to date. Here we report an affected individual presenting with a classic TRPSI phenotype who is heterozygous for a de novo intragenic $~ 36.3-\mathrm{kbp}$ duplication affecting exons 2-4 of TRPS1. Molecular analysis revealed the duplication to be in direct tandem orientation affecting the splicing of TRPS1. The aberrant transcripts are predicted to produce a truncated TRPS1 missing the nuclear localization signal and the GATA and IKAROS-like zinc-finger domains resulting in functional TRPS1 haploinsufficiency. Our study identifies a novel intragenic tandem duplication of TRPS1 and highlights the importance of molecular characterization of intragenic duplications.
\end{abstract}

[Supplemental material is available for this article.]

\section{INTRODUCTION}

Trichorhinophalangeal syndrome (TRPS) is a rare disorder, inherited in an autosomal dominant pattern, which is characterized by facial (bulbous nose, long philtrum, thin upper lip, and prominent ears), ectodermal (sparse, thin, and depigmented hair, thick eyebrows with lateral thinning, and dystrophic nails), and skeletal abnormalities (short stature, short hands and feet, hip dysplasia, and cone-shaped epiphyses at the phalanges) (Maas et al. 1993). Two distinct clinical types have been described for TRPS, with type I (TRPSI; OMIM\#190350) caused by pathogenic variants in TRPS1, and type II (TRPSII, also known as Langer-Giedion syndrome; OMIM\#150230). TRPSI is caused by loss of function mutations in TRPS1 and clinical features present with significant phenotypic variability among affected individuals and family members (Maas et al. 2015), including a form characterized by severe brachydactyly,

${ }^{6}$ Co-first authors 
previously proposed as a separate entity (TRPSIII; OMIM\#190351) (Niikawa and Kamei 1986; Lüdecke et al. 2001). TRPSII stems from contiguous gene deletions encompassing TRPS1, RAD21, and EXT1 (Maas et al. 1993). In addition to clinical features described in TRPSI, individuals with TRPSIl present with exostoses and an increased risk of intellectual disability (Langer et al. 1984).

TRPS1 (zinc-finger transcription factor TRPS1; OMIM\#604386) is a seven-exon transcriptional repressor involved in the regulation of chondrocyte and perichondrium development (Napierala et al. 2008). The most common pathogenic variants found in TRPS1 are nonsense and frameshift variants scattered along exons 4-7, which effectively truncate/alter the protein sequence (Maas et al. 2015). Intragenic and full gene deletions have also been reported with variable breakpoints, together with missense variants primarily clustering in exon 6 (Maas et al. 2015). In contrast to the more frequent deletions and single-nucleotide variants (SNVs), duplication events involving TRPS1 are rare and mostly involve single or few $(<10)$ nucleotides (Maas et al. 2015). Only a large pathogenic duplication of at least $600 \mathrm{bp}$ (c.2097-?_c.2700+?dup) in TRPS1 has been reported in an individual presenting with TRPSI, but no further characterization of this variant at the molecular or protein level has been reported (Maas et al. 2015). At the nucleotide level, duplication breakpoints could disrupt TRPS1 sequence or generate gene fusions, and when intragenic, they could give rise to new transcripts through splicing alterations or truncate proteins by introducing stop codons (Newman et al. 2015), all of which could contribute to TRPS pathogenesis.

In this report, we present an 11-yr-old male with a history of short stature, mild facial dysmorphism, and ectodermal and skeletal abnormalities consistent with a diagnosis of TRPSI. Chromosomal microarray analysis identified a de novo intragenic $~ 36.3-\mathrm{kbp}$ duplication affecting exons 2-4 of TRPS1 (NM_014112). Molecular characterization of the duplication by mate-pair sequencing (MPseq) revealed it to be in direct tandem orientation, and RNA sequencing (RNA-seq) discovered abnormal transcripts including the duplicated exons leading to out-of-frame products with predicted protein truncations. The present study is the first full characterization of an intragenic TRPS1 duplication leading to TRPSI and a valuable example for the study of intragenic tandem duplication effects in genomic transcriptional outputs.

\section{RESULTS}

\section{Case Presentation}

An 11-yr-old male (II-3, Fig. 1A) was referred for genetic evaluation because of a history of nonspecific skin hyperpigmentation involving left shoulder and axilla, short stature $(139.5 \mathrm{~cm}, 5.1 \%, Z=-1.63$ based on CDC length-for-age data), and scoliosis (Fig. 1B,C). He was mildly dysmorphic with a bulbous nose, prominent ears, a thin upper lip, and a long philtrum; his hair was noted to be fine and sparse, and his eyebrows were thick with lateral rarefaction. X-ray imaging revealed the presence of cone-shaped epiphyses at the phalanges (Fig. 1D,E). Similar skin lesions were present in his otherwise healthy mother. None of these features were present in his fraternal twin brother (II-4) or other full and half siblings (Fig. 1A).

\section{Genetic Testing}

A next-generation sequencing (NGS) RASopathy panel targeting 18 genes (NF1, SPRED1, LZTR1, PTPN11, PPP1CB, BRAF, CBL, HRAS, KRAS, NRAS, MAP2K1, MAP2K2, RAF1, RIT1, RASA2, SHOC2, SOS1, and SOS2) and deletion/duplication analysis of LZTR1, NF1, and SPRED1 (University of Alabama at Birmingham) was performed because of the proband's short stature and familial skin hyperpigmentation, the result of which was negative. 
A

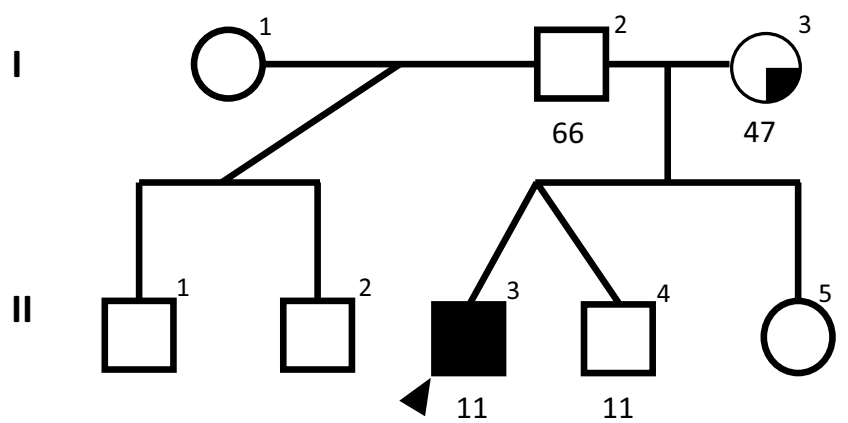

Short $\square \begin{aligned} & \text { Mild facial } \\ & \text { dysmorphism }\end{aligned}$ $\square$ Cone-shaped Skin dyspigmentation

B

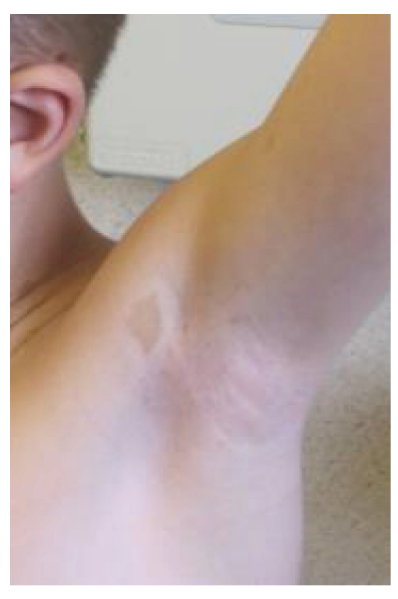

D

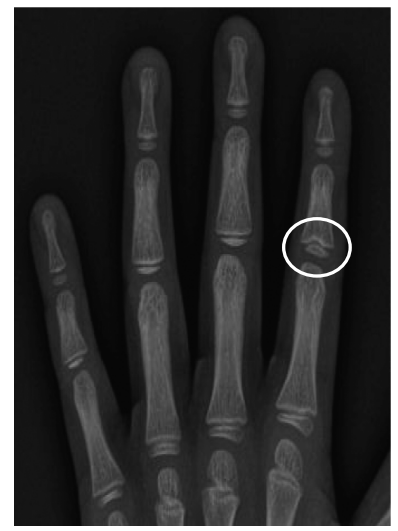

C

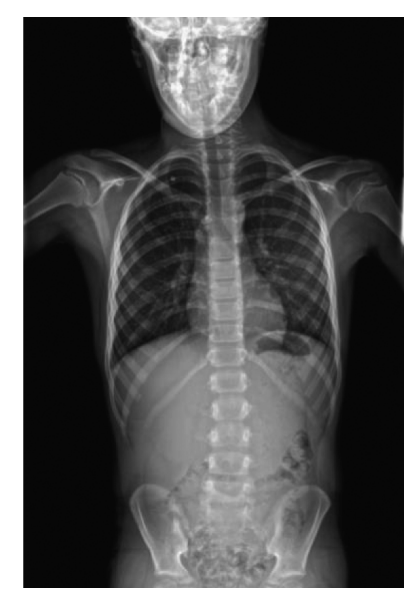

E

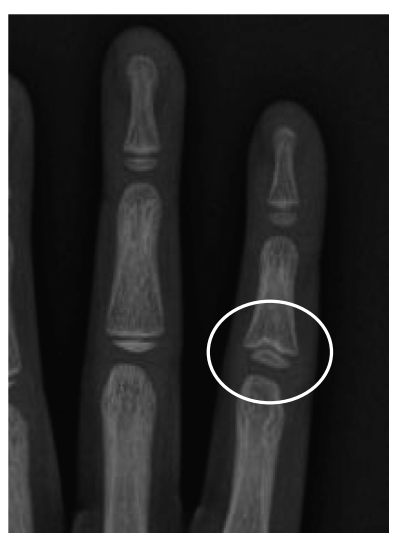

Figure 1. (A) Family pedigree for the analyzed proband. Proband is indicated with a black arrowhead. Numbers underneath indicate years of age. The clinical features of proband include $(B)$ skin hyperpigmentation, $(C)$ scoliosis, and $(D, E)$ cone-shaped epiphyses at the phalanges as indicated with the white circle ( $E$ is the zoom-in view of $D$ ).

Subsequent chromosome microarray (CMA) testing of the proband and his parents revealed the presence of a heterozygous 36.3-kbp duplication at 8q23.3 in the proband (arr[hg19] 8q23.3(116603667_116640019) × 3 dn), including exons 2-4 from TRPS1 (NM_014112) (Fig. 2A; Supplemental Fig. 1A,B). Parental CMA studies demonstrated that this duplication 
A TRPS1 exons (NM_014112)

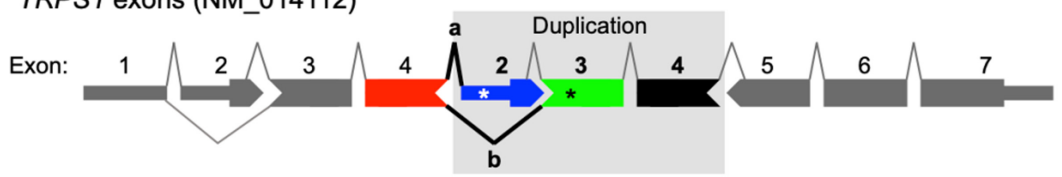

B TRPS1 domains (NM_014112)

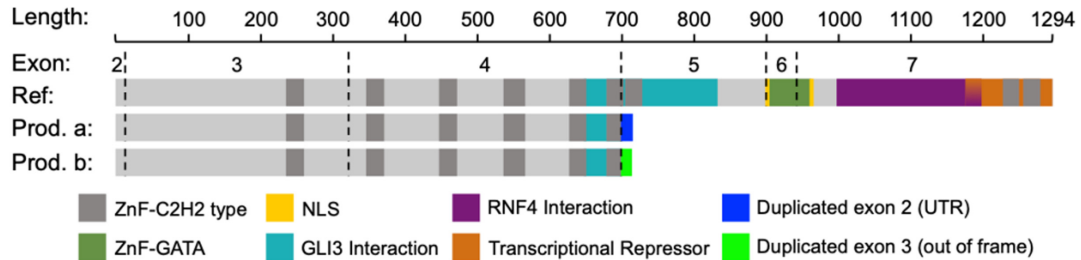

\section{Product a}

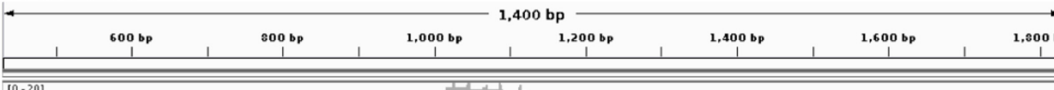
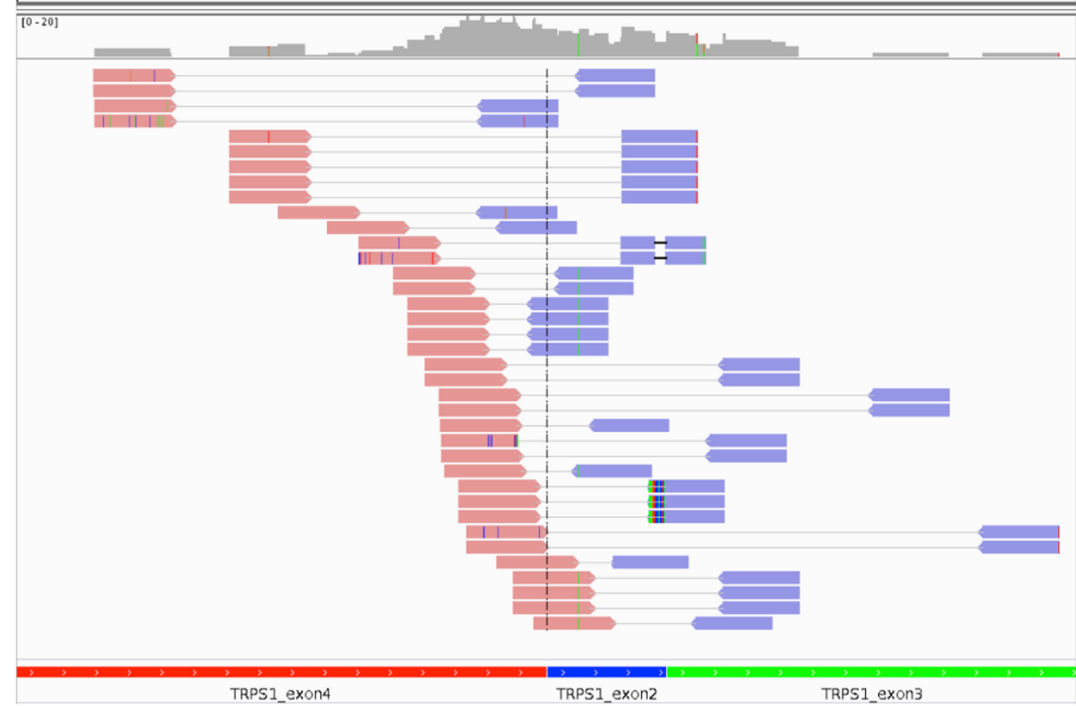

\section{Product b}

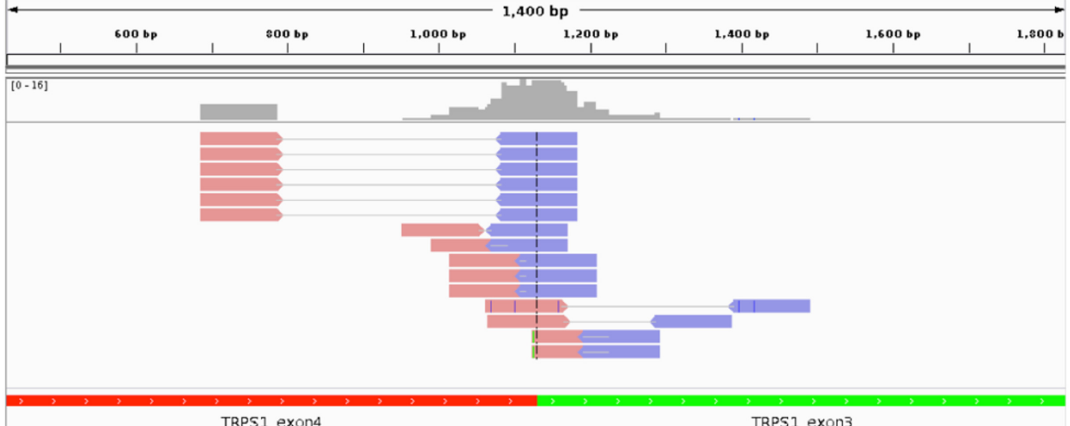

Figure 2. Abnormal transcripts associated with intragenic duplication in TRPS1. (A) Schematic of TRPS1 (NM_014112) with tandem duplication of exons 2-4 as found in proband. The observed splicing patterns are shown by lines connecting the exons, and abnormal splicing is shown in bold black lines. Asterisks indicate premature termination codons caused by abnormal splicing in duplicated region. (B) Schematic of TRPS1 with reported domains for the reference protein and for the predicted truncated proteins from each abnormal splicing event (product $a$ and b from A). Integrative Genomics Viewer (IGV) screenshots of the RNA-seq paired reads supporting abnormal splicing aligned to the proband-specific synthetic reference sequence of $(C)$ product a: exons 4-2-3, and (D) product b: exons 4-3 without intronic sequence included. 


\begin{tabular}{|c|c|c|c|c|c|c|c|c|}
\hline Gene & Chromosome & HGVS variant & $\begin{array}{l}\text { Variant } \\
\text { type }\end{array}$ & $\begin{array}{l}\text { Predicted } \\
\text { effect } \\
\text { (ACMG) }\end{array}$ & $\begin{array}{c}\text { MPseq } \\
\text { coverage }\end{array}$ & $\begin{array}{c}\text { Genotype } \\
\text { (heterozygous/ } \\
\text { homozygous) }\end{array}$ & $\begin{array}{l}\text { Parent } \\
\text { of origin }\end{array}$ & Comments \\
\hline TRPS1 & 8 & $\begin{array}{l}\text { GRCh38 } \\
\text { g.115591769_- } \\
115628731\end{array}$ & Duplication & $\begin{array}{l}\text { Likely } \\
\text { pathogenic }\end{array}$ & $24 \times$ & Heterozygous & De novo & $\begin{array}{l}\text { Variant was } \\
\text { confirmed by } \\
\text { Sanger }\end{array}$ \\
\hline
\end{tabular}

was not inherited from either parent, and it was therefore classified as a de novo event. Given the overlap between the proband's phenotype and TRPSI, it was hypothesized that the duplication could result in the functional disruption of the duplicated allele. To distinguish between a tandem and an insertional duplication, we analyzed the proband's blood using MPseq, a specialized NGS technique for the detection of structural rearrangements and copy-number abnormalities (Drucker et al. 2014; Johnson et al. 2018; Smadbeck et al. 2018). MPseq established the duplication to be in direct tandem orientation within TRPS1 (Supplemental Fig. 1C,D), with estimated breakpoints at Chr 8:115,591,898-115,628,547 (hg38). The identified MPseq duplication breakpoints were confirmed with Sanger sequencing and mapped to Chr 8:115,591,769-115,628,731 (hg38) (Table 1). Analysis of the Sanger sequence revealed microhomology between the rearranged segments and at the breakpoint junction, with a nearby sequence corresponding to transposon repetitive elements (SINE and hAT-Charlie) (Supplemental Data).

\section{Functional Studies}

To assess the functional impact of the intragenic duplication on TRPS1 transcription, we performed NGS whole-transcriptome sequencing from blood RNA. The schematic of the duplicated exons is shown in Figure 2A and protein domains in Figure 2B. We observed in proband's sample and unrelated samples (data not shown) skipping of exon 2, which occurs in a known transcript (NM_001282902) that is lowly expressed in the GTEx data (www .gtexportal.org). We created a proband-specific coding reference including exons 4-2-3 consecutively together and another reference for exons 4-3. By aligning the RNA-seq reads to the synthetic reference sequences (Fig. 2C,D, respectively), the reads supporting these aberrant junctions caused by the duplication event in proband can be visualized. A total of 52 read pairs support splicing from exon 4 to either exon 2 or 3 , with 13 of those having one read in the pair spanning the exon 4-2 junction and 15 having a one read in the pair spanning the exon 4-3 junction. The consequence to the transcript in either event is a premature termination codon (Fig. 2A). Exon 2 has 121 bp of 5' UTR and when splicing from exon 4, translates to 16 amino acids followed by a premature termination codon. When splicing from exon 4 to 3 , the transcript is out of frame and translates to 14 amino acids and a premature termination codon in the duplicated exon 3. At the exon level, reads per kilobase of transcript per million mapped reads (RPKM) revealed a higher number of reads mapping to exons 2-4 compared to a cohort of 127 differently affected individuals or unaffected relatives of subjects with suspected monogenetic disease (Fig. 3). An expression analysis using OUTRIDER (Brechtmann et al. 2018) showed only a modest increase in expression for TRPS1 (0.38 $\log _{2}$ fold change), most likely owing to the presence of the third copy of exons 2-4 ( 50\% of TRPS1 nucleotides) being transcribed and leading to additional reads contributing to overall gene expression. However, the nonduplicated exons in TRPS1 support normal expression levels and suggest the abnormal transcript with the duplicated exons may be escaping nonsense-mediated decay (NMD) that would be predicted from the premature termination codons. Given the proband's phenotype and the derived functional 

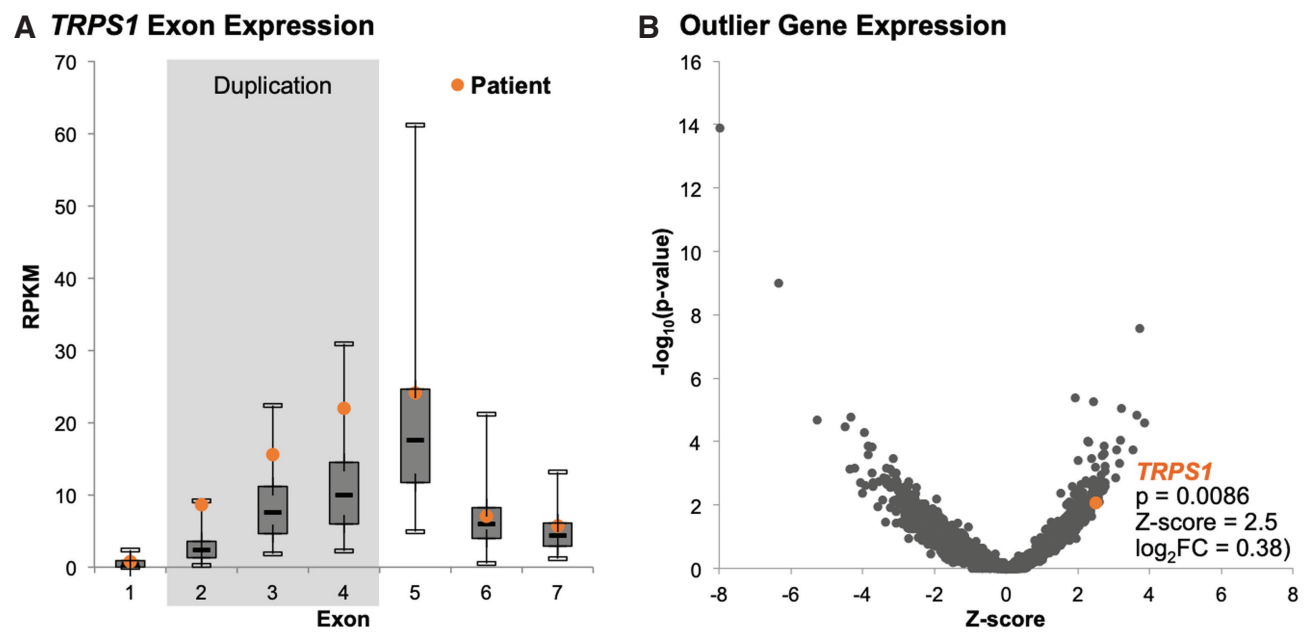

Figure 3. Gene expression analysis. (A) TRPS1 exonic expression levels in blood RNA of the proband compared to a cohort of 127 individuals differently affected with, or unaffected individuals related to individuals with, suspected rare monogenetic disease. (B) Gene expression changes called by OUTRIDER in this individual compared to the cohort represented as $-\log _{10}$ (unadjusted $P$-value) versus $Z$-score.

evidence of the intragenic duplication effect on the TRPS1 transcript, the proband was diagnosed with TRPSI.

\section{DISCUSSION}

We described a novel 36.3-kbp intragenic duplication in TRPS1 in an individual presenting with the classic ectodermal, facial, and skeletal abnormalities of TRPSI. CMA and MPseq analyses showed the duplication to be de novo and in direct tandem orientation within TRPS1 in the affected subject, effectively duplicating exons 2-4. Although more than 130 pathogenic variants have been reported in TRPS1, to our knowledge, this is the first characterization of a large intragenic duplication in TRPS1 associated with TRPSI.

The clinical features of TRPSI are caused by haploinsufficiency of TRPS1, a transcriptional repressor involved in the regulation of chondrocyte and perichondrium development. The identified intragenic duplication of exons $2-4$ was shown to produce abnormal transcripts with splicing patterns including exons 4-2 and 4-3. Both of these abnormal transcripts are predicted to (1) undergo NMD or (2) produce a likely nonfunctional protein missing the GATA zinc finger, the IKAROS-like zinc finger, and the nuclear localization signal (NLS) (Fig. 2B). In a NMD scenario, a lower-than-average number of mapped reads was expected for the transcripts. RPKM values of the duplicated exons were higher compared to normal male controls, whereas nonduplicated exons had RPKM ranges similar to controls. Such observations suggest that the aberrant intragenic duplication transcripts may not undergo NMD; further inspection of RNA-seq data failed to reveal any informative sequence variants for detecting allelic expression bias that would result from one transcript undergoing NMD. Either the allele with the duplicated exons is undergoing NMD, but overall gene transcription is up-regulated to compensate, or the abnormal transcripts escape NMD, are expressed at normal levels, and are translated into truncated nonfunctional protein lacking the functional GATA and IKAROS-like zinc fingers necessary for its correct genomic binding and repression activities. Such truncations can effectively cause functional haploinsufficiency of TRPS1 
in the reported individual and thus be a cause of TRPSI. Future proteomic experiments could evaluate the presence of truncated versions lacking the aforementioned domains.

Altogether, intragenic duplications within TRPS1 can have major clinical significance as illustrated in the currently studied individual. Identification and molecular characterization of other intragenic TRPS1 duplications will be necessary to assess genotype-phenotype correlations and to discover specific sites within the gene that are prone to further or recurrent chromosome rearrangements, as seen from our discovery of microhomology at the reported duplication junction. Microhomology-mediated mechanisms such as fork stalling and template switching (FoSTeS) (Lee et al. 2007) and microhomology-mediated break-induced replication (MMBIR) (Hastings et al. 2009) have been extensively associated with genomic germline duplications and deletions and other complex rearrangements. Both FoSTeS and MMBIR are plausible mechanisms to explain the origin of the intragenic TRPS1 duplication reported herein, given the detected junction microhomology and the presence of transposon sequences (SINE and hAT-Charlie) in the vicinity of the breakpoints, which can prime replication strand invasions upon double-strand breaks.

As we hope to have illustrated with this study, comprehensive molecular characterization of intragenic duplications is essential for the investigation of their pathogenicity and estimating potential recurrence risks. As NGS becomes a routine clinical test in clinical diagnosis, we surmise that additional intragenic duplications involving TRPS1 and many more genes will be uncovered, and their impact on protein structure assessed and correlated to diverse forms of human disease.

\section{MATERIALS AND METHODS}

Approval for this study was received by the Mayo Clinic Institutional Review Board, application number 12-009346; participants were consented for release of data included in this study. Chromosome microarray was performed on peripheral blood using the CytoScan HD Suite (Thermo Fisher Scientific), which evaluates allelic and copy-number information, according to the manufacturer's protocol. CMA data was analyzed using ChAS software version 3.1. MPseq was performed on DNA extracted from peripheral blood using AutoPure LS (QIAGEN). MPseq libraries were prepared using Nextera Mate Pair Library Preparation Kit (Illumina) and subsequently purified and processed for short-read library preparation using TruSeq DNA Library Prep kit (Illumina). Purified libraries were sequenced in an Illumina HiSeq 2500 using RapidRun mode to obtain 101-bp reads. MPseq data was processed with BIMAv3 and analyzed with SVAtools version 0.24.9. Polymerase chain reaction (PCR) experiments were performed to amplify breakpoint junctions identified by MPseq.

RNA was isolated using the miRNeasy Mini Kit (QIAGEN) following the standard protocol from blood drawn in a PAXgene Blood RNA Tube (QIAGEN). RIN and DV200 values were determined for starting RNA concentrations using the Agilent Bioanalyzer or TapeStation. RNA libraries were prepared according to the manufacturer's instructions for the TruSeq RNA Access Library Prep Kit (Illumina). Coding regions of the transcriptome were captured by pooling four of the cDNA libraries at $200 \mathrm{ng}$ each following the manufacturer's instructions for the TruSeq RNA Access Library Prep Kit (Illumina). The concentration and size distribution of the final libraries were determined on an Agilent Bioanalyzer DNA 1000 chip (Agilent Technologies). A final quantification, using Qubit fluorometry (Invitrogen), was performed to confirm sample concentration.

Libraries were sequenced at $\sim 65$ million fragment reads per sample (4 samples/lane) following Illumina's standard protocol using the Illumina cBot and HiSeq 3000/4000 PE Cluster Kit. The flow cells were sequenced as $100 \times 2$ paired-end reads on an Illumina HiSeq 4000 
using HiSeq 3000/4000 sequencing kit and HCS v3.3.20 collection software. Base calling was performed using Illumina's RTA version 2.5.2.

RNA-sequencing analysis was performed using MAP-RSeq (Kalari et al. 2014). Reads were aligned to the human genome (hg19) and transcriptome using TopHat2 (Kim et al. 2013) running Bowtie (v1) (Langmead 2010). Gene- and exon-level read counts were generated using HiSeq (Anders et al. 2015) and BEDTools (Quinlan 2014), respectively.

Custom reference creation: Two custom human reference sequences were constructed to represent the aberrant transcripts arising from the tandem duplication of TRPS1 exons 2-4. Constituent exon sequences were extracted from the UCSC hg19 FASTA sequence utilizing genomic coordinates for RefSeq transcript NM_014112.4. FASTA sequence for the exons were concatenated in the order Exon $4 \rightarrow$ Exon $2 \rightarrow$ Exon 3 and Exon $4 \rightarrow$ Exon 3 in the $5^{\prime}$ to $3^{\prime}$ direction, mimicking the expected aberrant juxtaposition of the exons in the final transcripts. All other TRPS1 exons were excluded from the custom reference to ensure specificity of alignments supporting the aberrantly formed splice junctions. Alignment: Cutadapt (Martin 2011) was used to remove poly(A) tails from raw RNA-seq reads. Alignment to the custom reference sequence was performed using BWA MEM (Li 2013) with default parameters. SAMTools (Li et al. 2009) was used to remove reads with multiple mappings or improper read-pairing or to insert sizes varying from the expected range. Read pairs containing one mate crossing an aberrantly formed splice junction by fewer than 10 bases were filtered to prevent nonspecific alignments that might align with equal homology with the normal transcript. Integrative Genomics Viewer (IGV) (Thorvaldsdóttir et al. 2013) was used to generate snapshots of the final alignments. A custom annotation track was created to enable identification of exon order in the custom reference.

Outlier expression analyses: Expression counts and RPKMs were computed as above for a cohort of 128 samples from the Mayo Clinic Service Line 2 for rare and undiagnosed diseases. Genes with low expression in blood were filtered out. Specifically, genes whose 0.95 quantile FPKM was $<1$ or whose counts were 0 in more than one-quarter of the samples were excluded from the analysis. Outlier expression analysis was then performed using OUTRIDER (Brechtmann et al. 2018) with the Benjamini-Hochberg correction for multiple comparisons, which provided confounder-corrected $z$-scores, $\log _{2}$ fold changes, $P$-values, and adjusted $P$ values.

\section{ADDITIONAL INFORMATION}

\section{Data Deposition and Access}

The duplication position within TRPS1 was submitted to the Leiden Open Variation Database (Fokkema et al. 2011) under accession number 0000597835. Patient consent does not allow for deposition of raw data.

\section{Ethics Statement}

The studied family was counseled and consented under IRB 12-009346 from the Mayo Clinic Department of Laboratory Medicine and Pathology and Center for Individualized Medicine.

\section{Acknowledgments}

We thank the reported family for their enthusiasm and participation in this research report. We also thank the staff at Mayo Clinic Genomics Laboratory for their excellence in the clinical processing and testing of the samples from this family. 
Competing Interest Statement

The authors have declared no competing interest.

\section{Referees}

Scott E Hickey

Anonymous

Received July 30, 2019; accepted in revised form October 1, 2019.

\section{Author Contributions}

C.J.Z.-M. and M.A.C. performed experiments and interpreted data; M.A.C., S.B., G.J., and G.O. analyzed RNA-seq data; S.T.P. and D.B.-V. provided clinical interpretations and consultation; L.A.B. interpreted CMA data; all authors read and approved the manuscript.

\section{Funding}

Funding was provided by the Mayo Clinic Department of Laboratory Medicine and Pathology and Center for Individualized Medicine.

\section{REFERENCES}

Anders S, Pyl PT, Huber W. 2015. HTSeq-a Python framework to work with high-throughput sequencing data. Bioinformatics 31: 166-169. doi:10.1093/bioinformatics/btu638

Brechtmann F, Mertes C, Matusevičiūtè A, Yépez VA, Avsec Z, Herzog M, Bader DM, Prokisch H, Gagneur J. 2018. OUTRIDER: a statistical method for detecting aberrantly expressed genes in RNA sequencing data. Am J Hum Genet 103: 907-917. doi:10.1016/j.ajhg.2018.10.025

Drucker TM, Johnson SH, Murphy SJ, Cradic KW, Therneau TM, Vasmatzis G. 2014. BIMA V3: an aligner customized for mate pair library sequencing. Bioinformatics 30: 1627-1629. doi:10.1093/bioinformatics/ btu078

Fokkema IF, Taschner PE, Schaafsma GC, Celli J, Laros JF, den Dunnen JT. 2011. LOVD v.2.0: the next generation in gene variant databases. Hum Mutat 32: 557-563. doi:10.1002/humu.21438

Hastings PJ, Ira G, Lupski JR. 2009. A microhomology-mediated break-induced replication model for the origin of human copy number variation. PLoS Genet 5: e1000327. doi:10.1371/journal.pgen.1000327

Johnson SH, Smadbeck JB, Smoley SA, Gaitatzes A, Murphy SJ, Harris FR, Drucker TM, Zenka RM, Pitel BA, Rowsey RA, et al. 2018. SVAtools for junction detection of genome-wide chromosomal rearrangements by mate-pair sequencing (MPseq). Cancer Genet 221: 1-18. doi:10.1016/j.cancergen.2017.11.009

Kalari KR, Nair AA, Bhavsar JD, O'Brien DR, Davila JI, Bockol MA, Nie J, Tang X, Baheti S, Doughty JB, et al. 2014. MAP-RSeq: Mayo analysis pipeline for RNA sequencing. BMC Bioinformatics 27: 224. doi:10.1186/ 1471-2105-15-224

Kim D, Pertea G, Trapnell C, Pimentel H, Kelley R, Salzberg SL. 2013. TopHat2: accurate alignment of transcriptomes in the presence of insertions, deletions and gene fusions. Genome Biol 14: R36. doi:10.1186/gb2013-14-4-r36

Langer LO Jr, Krassikoff N, Laxova R, Scheer-Williams M, Lutter LD, Gorlin RJ, Jennings CG, Day DW. 1984. The tricho-rhino-phalangeal syndrome with exostoses (or Langer-Giedion syndrome): four additional patients without mental retardation and review of the literature. Am J Med Genet 19: 81-112. doi:10 .1002/ajmg.1320190110

Langmead B. 2010. Aligning short sequencing reads with bowtie. Curr Protoc Bioinformatics 32: 11.17.1111.17.14. doi:10.1002/0471250953.bi1107s32

Lee JA, Carvalho CM, Lupski JR. 2007. A DNA replication mechanism for generating nonrecurrent rearrangements associated with genomic disorders. Cell 131: 1235-1247. doi:10.1016/j.cell.2007.11.037

$\mathrm{Li} \mathrm{H.} \mathrm{2013.} \mathrm{Aligning} \mathrm{sequence} \mathrm{reads,} \mathrm{clone} \mathrm{sequences} \mathrm{and} \mathrm{assembly} \mathrm{contigs} \mathrm{with} \mathrm{BWA-MEM.} \mathrm{arXiv} \mathrm{e-prints.}$

Li H, Handsaker B, Wysoker A, Fennell T, Ruan J, Homer N, Marth G, Abecasis G, Durbin R, 1000 Genome Project Data Processing Subgroup. 2009. The Sequence Alignment/Map format and SAMtools. Bioinformatics 25: 2078-2079. doi:10.1093/bioinformatics/btp352

Lüdecke HJ, Schaper J, Meinecke P, Momeni P, Gross S, von Holtum D, Hirche H, Abramowicz MJ, Albrecht B, Apacik C, et al. 2001. Genotypic and phenotypic spectrum in tricho-rhino-phalangeal syndrome types I and III. Am J Hum Genet 68: 81-91. doi:10.1086/316926

Maas S, Shaw A, Bikker H, Hennekam, RCM. 1993. Trichorhinophalangeal syndrome. In GeneReviews ${ }^{\circledR}$ (ed. Adam MP, Ardinger HH, Pagon RA, et al.). University of Washington, Seattle.

Maas SM, Shaw AC, Bikker H, Lüdecke HJ, van der Tuin K, Badura-Stronka M, Belligni E, Biamino E, Bonati MT, Carvalho DR, et al. 2015. Phenotype and genotype in 103 patients with tricho-rhino-phalangeal syndrome. Eur J Med Genet 58: 279-292. doi:10.1016/j.ejmg.2015.03.002

Martin M. 2011. Cutadapt removes adapter sequences from high-throughput sequencing reads. EMBnet.journal 17: 3. doi:10.14806/ej.17.1.200 
Napierala D, Sam K, Morello R, Zheng Q, Munivez E, Shivdasani RA, Lee B. 2008. Uncoupling of chondrocyte differentiation and perichondrial mineralization underlies the skeletal dysplasia in tricho-rhino-phalangeal syndrome. Hum Mol Genet 17: 2244-2254. doi:10.1093/hmg/ddn125

Newman S, Hermetz KE, Weckselblatt B, Rudd MK. 2015. Next-generation sequencing of duplication CNVs reveals that most are tandem and some create fusion genes at breakpoints. Am J Hum Genet 96: 208-220. doi:10.1016/j.ajhg.2014.12.017

Niikawa N, Kamei T. 1986. The Sugio-Kajii syndrome, proposed tricho-rhino-phalangeal syndrome type III. Am J Med Genet 24: 759-760. doi:10.1002/ajmg.1320240420

Quinlan AR. 2014. BEDTools: the Swiss-army tool for genome feature analysis. Curr Protoc Bioinformatics 47: 1-34. doi:10.1002/0471250953.bi1112s47

Smadbeck JB, Johnson SH, Smoley SA, Gaitatzes A, Drucker TM, Zenka RM, Kosari F, Murphy SJ, Hoppman N, Aypar U, et al. 2018. Copy number variant analysis using genome-wide mate-pair sequencing. Genes Chromosomes Cancer 57: 459-470. doi:10.1002/gcc.5

Thorvaldsdóttir H, Robinson JT, Mesirov JP. 2013. Integrative Genomics Viewer (IGV): high-performance genomics data visualization and exploration. Brief Bioinform 14: 178-192. doi:10.1093/bib/bbs017 


\section{COLD SPRING HARBOR Molecular Case Studies}

\section{An intragenic duplication of TRPS1 leading to abnormal transcripts and causing trichorhinophalangeal syndrome type I}

Cinthya J. Zepeda-Mendoza, Margot A. Cousin, Shubham Basu, et al.

Cold Spring Harb Mol Case Stud 2019, 5: a004655 originally published online October 29, 2019

Access the most recent version at doi: $10.1101 / \mathrm{mcs} .0004655$
Supplementary http://molecularcasestudies.cshlp.org/content/suppl/2019/11/07/mcs.a004655.D Material C1
License This article is distributed under the terms of the Creative Commons Attribution-NonCommercial License, which permits reuse and redistribution, except for commercial purposes, provided that the original author and source are credited.
Email Alerting Receive free email alerts when new articles cite this article - sign up in the box at the Service top right corner of the article or click here.

